os s i er

$>$ La fiscalité locale:

regards comparatifs

Colloque de Lyon

10 octobre 2019

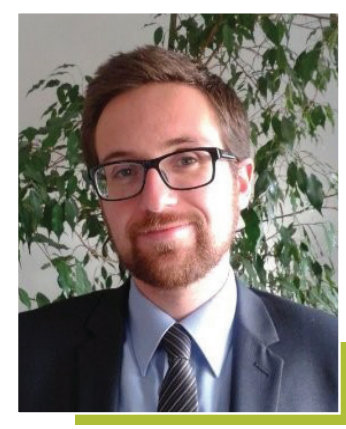

Cédric MEURANT

Maître de conférences en droit public, Université Jean Moulin Lyon 3,

Équipe de droit public de Lyon (EA 666)

\title{
Le contentieux de la fiscalité locale entre personnes publiques
}

\author{
Mol5-C|éS : fiscalité locale - relations entre l'État et les collectivités territoriales - \\ établissements publics de coopération intercommunale - contentieux
}

\begin{abstract}
Les tensions fiscales entre l'État, les collectivités territoriales et les $\mathrm{EPCI}$ nées de la décentralisation et de la recentralisation peuvent se traduire par des contentieux juridictionnels. Leur étude permet l'analyse de ces discordes et pose la question de savoir quelle évolution le juge fait prévaloir : la décentralisation ou la recentralisation?
\end{abstract}

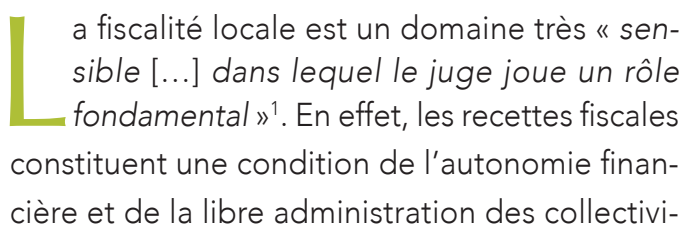

\footnotetext{
${ }^{1}$ Constat formulé à propos des relations financières: X. Cabannes, « Le contentieux des relations financières entre l'État et les collectivités territoriales », AJDA 2016, p. 598.

${ }^{2}$ Art. $72-2$ de la Constitution. Le législateur n'a pas I'obligation de laisser cette faculté aux collectivités : C.C., 6 mai 1991, Loi instituant une dotation de solidarité urbaine, n 91-291 DC, §. 29.

${ }^{3}$ Ainsi que l'affirme la Deuxième partie du CGI.

${ }^{4}$ Si ces impôts entrent dans le champ de l'art. LO 1114-2 CGCT, il est régulièrement proposé de les exclure du calcul de la "part déterminante des ressources »: C. Jerretie, A. Viala, Mission flash sur la réforme des institutions. Expérimentation territoriale. Autonomie financière des collectivités territoriales. Rapport $n^{\circ} 912$, Délégation aux collectivités territoriales et à la décentralisation, Assemblée nationale, 2019, p. 67.

5 B. Faure, Droit des collectivités territoriales, 5éd., Dalloz, 2018, Précis, p. 660.

${ }^{6}$ C.C., 29 déc. 2009, Loi de finances pour 2010, n²009-599 DC, §. 64 et 94.

Art. $72-2$ de la Constitution; art. 1636 B sexies CGI.

${ }^{8}$ Art. 1er de la loi n $80-10$ du 10 janvier 1980 portant aménagement de la fiscalité directe locale.
}

tés territoriales puisqu'elles doivent représenter " une part déterminante de l'ensemble de leurs ressources $»^{2}$. Si la fiscalité locale est usuellement définie comme groupant les impositions "perçues » par les collectivités territoriales ${ }^{3}$, elle ne comprend strictement pas les impôts nationaux partagés par l'État ${ }^{4}$, mais seulement ceux sur lesquels elles peuvent exercer un « pouvoir fiscal propre $»^{5}$. À défaut d'une véritable «autonomie fiscale $"^{6}$, les collectivités territoriales peuvent être habilitées par le législateur à fixer l'assiette et/ou le taux d'imposition des impôts locaux ${ }^{7}$. Dès lors, la fiscalité locale regroupe les quatre impôts directs locaux ${ }^{8}$ que sont les taxes foncières, d'habitation et la contribution économique territoriale (CET), ainsi qu'une myriade de prélèvements, directs ou indirects, obligatoires 
ou facultatifs, figurant dans le Code général des impôts (CGI)9, le Code général des collectivités territoriales (CGCT) ${ }^{10}$, le Code de l'Urbanisme ${ }^{11}$ ou encore celui du Patrimoine ${ }^{12}$.

Cette fiscalité locale symbolise l'esprit de la décentralisation française : I'autonomie laissée aux collectivités territoriales n'est constituée que de «permissions législatives » accordées par l'État ${ }^{13}$ qui exerce donc un contrôle important sur la gestion de ces impôts. Ainsi, ce sont ses services qui en assurent l'assiette, le contrôle et le recouvrement ${ }^{14}$. De même, il reverse aux collectivités territoriales à la fin de chaque mois un douzième du produit de ces impositions ${ }^{15}$ qui, dans l'attente, est affecté au programme $n^{\circ} 833$ « Avances sur le montant des impositions revenant aux départements, communes, établissements et divers organismes » du compte de concours financier «Avances aux collectivités territoriales». Cette maîtrise étatique de la fiscalité locale progresse aujourd'hui, au point qu'une renationalisation de ces impositions est évoquée. Ainsi, le contribuable national se "substitue au contribuable local $»^{16}$ puisque les collectivités territoriales se voient de plus en plus transférer le produit d'impôts partagés sur lesquels elles n'ont aucun pouvoir ${ }^{17}$.

De plus, un second mouvement de recentralisation de la fiscalité locale entre les mains des établissements publics de coopération intercommunale (EPCI) à fiscalité propre est aujourd'hui à I'œuvre avec le développement à marche forcée de cette association qui conduit les communes à leur abandonner une fraction de leurs recettes fiscales. Ainsi, les quatre types de groupement de communes à fiscalité propre (communautés de communes, d'agglomération, urbaine et métropoles) disposent d'un pouvoir fiscal ${ }^{18}$ qui, schématiquement, peut prendre trois formes: d'abord, la CET unique qui autorise l'EPCI à percevoir et à moduler l'intégralité de cet impôt sur son territoire ; ensuite, la fiscalité additionnelle aux quatre impôts directs locaux qui lui permet de recevoir directement une part supplémentaire à ces impositions qui s'ajoute à celle perçue par les communes; enfin, la CET de zone qui unifie à son bénéfice la gestion et la perception de cet impôt pour une zone d'activité économique ${ }^{19}$. Inversement, les EPCl sans fiscalité propre (les syndicats de communes) ne peuvent pas disposer d'un pouvoir fiscal dérivé : si la contribution budgétaire versée par les communes membres pour abonder leurs budgets peut être fiscalisée ${ }^{20}$, cela ne leur laisse aucun pouvoir de fixation des taux ou de l'assiette ${ }^{21}$.
Ces deux dynamiques de recentralisation durcissent les relations qui existent entre les trois principales ${ }^{22}$ personnes publiques actrices de la fiscalité locale : les collectivités territoriales, les $\mathrm{EPCl}$ et l'État. Des contentieux juridictionnels entre ces personnes en résultent logiquement et s'ajoutent à ceux, classiques, nés de la décentralisation et de l'autonomie qu'elle accorde ${ }^{23}$. Ils sont principalement tranchés par le juge administratif dès lors que la fiscalité locale est surtout composée d'impositions directes ${ }^{24}$. Le juge judiciaire est subsidiairement compétent pour les quelques impositions locales indirectes ${ }^{25}$. De même, l'intervention du Conseil constitutionnel est parfois sollicitée. Dans ce cadre, ces contestations font l'objet de quelques adaptations procédurales. Par exemple, dans le cadre « d'un litige relatif à la répartition des ressources financières publiques entre personnes publiques », les collectivités territoriales ont une liberté d'argumentation limitée puisqu'elles ne peuvent pas invoquer de moyens tirés de la violation des stipulations de la Convention européenne de sauvegarde des droits de l'homme et des libertés fondamentales, et notamment de l'article $1^{\text {er }}$ de son Premier protocole additionnel ${ }^{26}$. Certaines spécificités sont ensuite propres au contentieux de la fiscalité locale : s'il présente peu de particularités par rapport aux règles singulières du contentieux fiscal ${ }^{27}$, ces litiges sont, notamment, réglés par un juge unique ${ }^{28}$, en dernier ressort ${ }^{29}$.

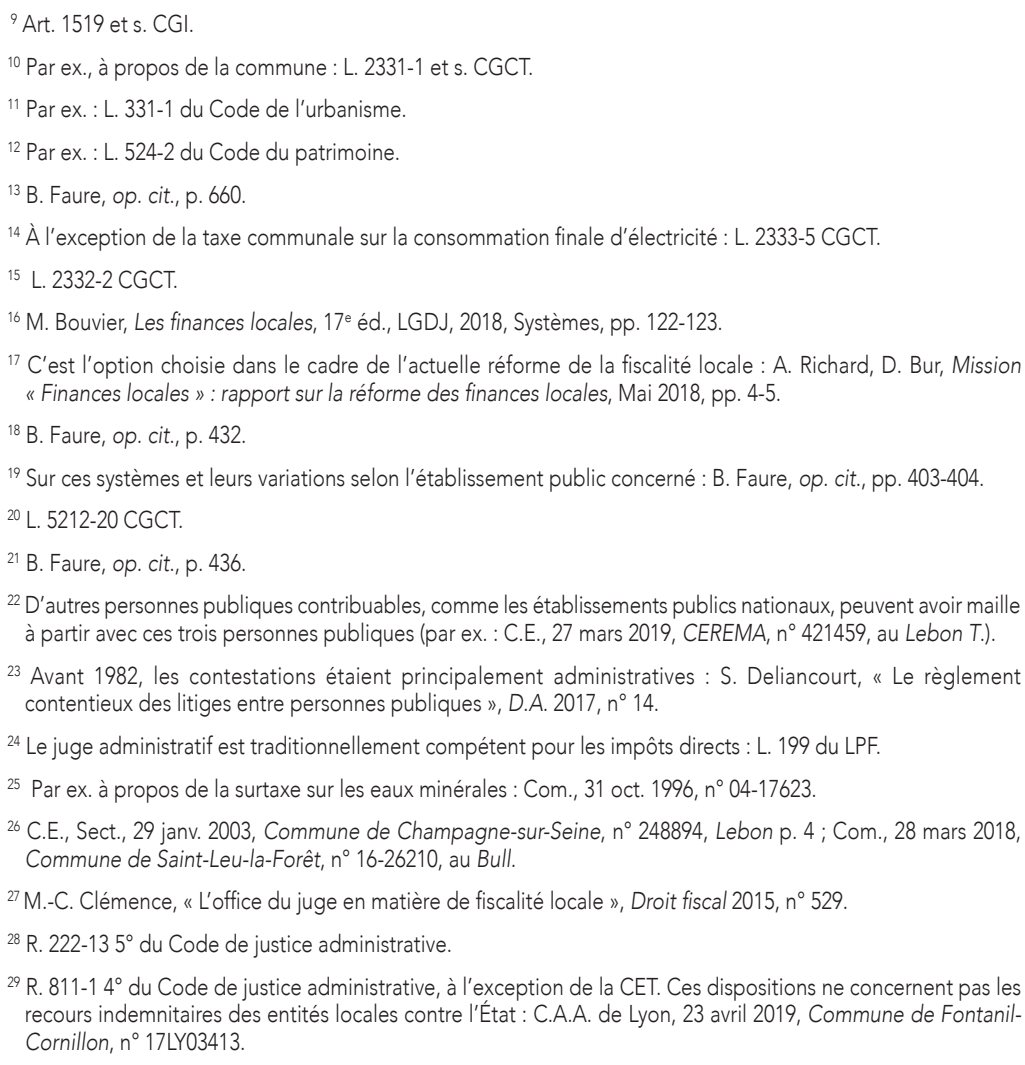


O s s i e r

$>$ La fiscalité locale : regards comparatifs Colloque de Lyon 10 octobre 2019

${ }^{30}$ Exposant les subtilités de cette jurisprudence : M.-C. Clémence, loc. cit., $n^{\circ} 529$.

${ }^{31} \mathrm{M}$. Bouvier, «De l'autonomie financière et de la libre administration des collectivités territoriales " JCP-A 2002, n' 1097.

32 J. Carbonnier, Sociologie du droit. Sociologie du droit de la famille, 1963, p. 19

${ }^{33}$ S. Deliancourt, loc. cit., $n^{\circ} 14$.

${ }^{34}$ C.E., Sect., 27 fév. 2004, Popin, $n^{\circ} 217254$, Lebon p. 86.

35 B. Delaunay, «Les litiges financiers et fiscaux entre personnes publiques », D.A. 2017, nº 10 .

${ }^{36}$ B. Faure, op. cit., p. 666

${ }^{37}$ M. Bouvier, M.-C. Esclassan J.-P. Lassale, Finances publiques, $11^{\circledR}$ éd., LGDJ 2012, Manuels, p. 937.

${ }^{38}$ P. Moulié, L'imposition des personnes publiques, Préface de P. Lalumière, LGDJ, 1972, BSF t. 9, pp. 24-26.

${ }^{39}$ Art. 1654 CGI

${ }^{40}$ Comp. : C.E., 16 mai 2007 Communauté de communes du Pays de LanderneauDaoulas, $n^{\circ} 272727$, Lebon T. p. 798 ; C.E., 22 sept. 2014, Ministre délégué chargé du Budget, $n^{\circ} 360742$, Lebon T. p. 620. Retraçant cette évolution : J. Lamarque O. Négrin, L. Ayrault, Droit fiscal général, $4^{e}$ éd. Lexisnexis, 2016, Manuels, pp. 767-768.

41 J.-L. Albert, Fiscalité et personnes publiques, LGDJ, 2012, Systèmes, pp. 114 et 127

${ }^{42} \mathrm{C}$. Louit, " Retour sur une guerre picrocholine : I'État français applique-t-il sa propre loi ? ", Droit fiscal 2007, nº 784.

${ }^{43}$ Réponse de Mme A.-M. Idrac, J.O. Sénat, 14 janv. 2009, p. 233.

${ }^{44}$ C.E., 6 nov. 2009, Ministre de la Défense, $n^{\circ} 310642$.

${ }^{45}$ Art. $72-5$ de la Constitution.

${ }^{46} \mathrm{C}$. Delivre-Gilg, «Les relations financières entre collectivités territoriales », in S. Caudal, F. Robbes, Les relations entre collectivités territoriales, L'Harmattan, 2005, Logiques juridiques, p. 174.

${ }^{47} \mathrm{M}$. Collet, Finances publiques, LGDJ, 2016, Domat, p. 352

${ }^{48}$ C.C., 6 juin 2014, Commune de Guyancourt, n²014-397 DC.

${ }^{49}$ C.A.A. de Lyon, 16 juin 2018, Communauté de communes du Pays de Saint-Galmier, n० 16 LY02634.

${ }^{50}$ Sur leur nature particulière : C.E., 14 oct. 2005, Commune de Pagny-Moselle, $n^{\circ} 255179$ Lebon T. p. 754

Si ce juge écarte comme irrégulière la méthode d'évaluation de la valeur locative des biens retenue par l'administration, il a alors le devoir juridictionnel de la déterminer d'office ${ }^{30}$.

L'existence de ces contentieux démontre que le juge est considéré par l'État, les collectivités territoriales et les EPCl comme l'arbitre des intérêts publics dont ils ont respectivement la charge. II est aussi regardé par les personnes publiques victimes de la recentralisation de la fiscalité locale comme le moyen de résister à ce transfert et de préserver leur pouvoir politique et leur autonomie de gestion, garantis par leur capacité fiscale ${ }^{31}$. Par conséquent, si ces litiges ne constituent que la "pathologie de la réalité sociale $»^{32}$

des relations entre l'État, les collectivités territoriales et les $\mathrm{EPCl}$, ils reflètent dans le prétoire les tensions nées de la décentralisation et de la recentralisation. Ils en permettent ainsi I'analyse ${ }^{33}$ et posent plus fondamentalement la question de savoir quelle dynamique le juge, qui statue au nom de l'État ${ }^{34}$, fait prévaloir. Certes, cette recherche est compliquée par le fait que ces litiges trouvent parfois une solution amiable et secrète $^{35}$. Mais l'accroissement des contentieux en la matière rend possible une étude jurisprudentielle. Ainsi, I'analyse permet de constater que les contentieux entre personnes publiques liés à l'organisation originelle de la décentralisation existent toujours (1.), et que ceux qui tiennent aux recentralisations se multiplient (2.).

\section{La pérennité des contentieux nés du pouvoir fiscal des entités locales}

La décentralisation du pouvoir fiscal date de la loi du 10 janvier 1980 aménageant la fiscalité locale. Puis les actes «I » et « I| » vont fonder la décentralisation sur une tension entre, d'une part, l'autonomie de gestion et financière des entités locales, d'autre part, le contrôle exercé par l'État sur elles. Cette organisation va mécaniquement créer des litiges liés à la fiscalité locale, qu'ils tiennent au premier pilier (A.), ou au second (B.).

\section{A. Les contentieux résultant de l'au- tonomie accordée aux entités locales}

La décentralisation attribue une autonomie administrative et financière aux collectivités territoriales qui s'exerce dans le carcan juridique dessiné par l'État. En matière fiscale, cette autonomie se traduit par la possibilité pour les collectivités territoriales "d'aménager la règle fiscale ${ }^{36}$ relative aux impôts locaux. Elle engendre deux types de contentieux entre personnes publiques. Les premiers opposent ces dernières, qui ont la qualité de contribuable local, à l'État, "fermier général » des entités locales bénéficiaires des impôts locaux ${ }^{37}$. Suivant le « principe de similitude ${ }^{38}$ des personnes publiques par rapport aux personnes privées en matière fiscale lorsqu'elles exercent des activités commerciales ${ }^{39}$, la qualité de contribuable local leur est reconnue. Mais elles ne l'admettent pas toujours facilement, ce qui génère de nombreux litiges, ainsi que l'illustre l'évolution bien connue du raisonnement du juge pour déterminer si les activités professionnelles non-salariées exercées habituellement par les personnes publiques sont assujetties à la taxe professionnelle ${ }^{40}$. En outre, le secteur militaire génère de nombreux contentieux ${ }^{41}$. D'autant plus que les entités bénéficiaires de l'impôt local ou les services étatiques chargés de l'établir peuvent éprouver des difficultés pour accéder aux informations relatives à ces activités pour établir l'imposition. Le secret-défense qui les entoure peut alors déséquilibrer ces procès au bénéfice de l'État ${ }^{42}$. Compte tenu du rôle qu'il joue dans le recouvrement de ces impôts, des tensions ${ }^{43}$ et des procès ${ }^{44}$ ont même pu survenir entre les ministres des Armées et du Budget.

En second lieu, les mécanismes de péréquation horizontale ont donné lieu à des contentieux horizontaux entre collectivités locales. Certains de ces dispositifs, qui sont « destinés à favoriser l'égalité entre les collectivités territoriales " $^{45}$, sont des "péréquations fiscales ${ }^{46}$ : ils " consistent à prélever une partie des ressources fiscales de certaines collectivités pour la reverser à des collectivités moins favorisées ${ }^{47}$. Ainsi, les collectivités bénéficiaires peuvent critiquer l'insuffisance de la somme qui leur est versée. Inversement, les collectivités qui souffrent de ces dispositifs mis en place par le législateur peuvent en contester la constitutionnalité ${ }^{48}$ ou leur contribution au fonds ${ }^{49}$.

De plus, des contentieux contractuels peuvent naître de l'exécution des conventions de péréquation permises par l'article 11 de la loi du 10 janvier $1980^{50}$ conclues entre départements ou régions, ou entre communes membres d'un EPCl qui gère une zone d'activités économiques dans laquelle sont relocalisées des entreprises situées 
jusqu'ici sur le territoire d'autres communes. Ces conventions organisent un " partage de fiscalité $»^{51}$ à propos, notamment, de la contribution économique territoriale. Ainsi, elles répartissent les produits de cette imposition qui « est perçue par une seule commune sur laquelle sont implantées les entreprises ». De nombreux contentieux sont nés de l'exécution de ces contrats, ainsi que l'illustrent les trois fameux arrêts Commune de Béziers ${ }^{52}$.

\section{B. Les contentieux résultant des contrôles de l'État}

Dans l'organisation décentralisée française, I'autonomie des collectivités territoriales et des EPCl, est encadrée par une série de contrôles étatiques qui garantissent « le respect des lois » ${ }^{53}$ par ces derniers. Ces vérifications entraînent deux types de contentieux dans le cadre de la fiscalité locale entre, d'une part, l'État, d'autre part, les entités locales. Tout d'abord, le représentant de l'État peut, lorsqu'il constate une violation des règles relatives à la fiscalité locale dans le cadre de son contrôle de la légalité, déférer au tribunal administratif les modalités conventionnelles de répartition du produit de la fiscalité locale entre les communes et les $\mathrm{EPCl}^{54}$, le régime fiscal défini par les collectivités régies par l'article 74 de la Constitution ${ }^{55}$ ou, très classiquement, les délibérations de ces entités locales fixant les taux et l'assiette des impositions directes locales. Dans le cadre de ce dernier contentieux, le préfet ne peut pas invoquer une exception d'illégalité tirée de ce qu'une délibération fixant ces taux pour un exercice fiscal est irrégulière dès lors que la délibération ayant le même objet pour l'exercice fiscal précédent était elle-même illégale. En effet, le premier acte $n^{\prime}$ est pas une mesure d'application du second ${ }^{56}$. Mais, compte tenu des dispositions spécifiques encadrant le vote du taux de l'ancienne taxe professionnelle, l'annulation d'une délibération en fixant le taux au titre d'une année fiscale est de nature à entraîner l'annulation « par voie de conséquence » de la délibération de l'année qui suit et qui a le même objet ${ }^{57}$. De plus, la collectivité territoriale ou l'EPCI qui subit l'annulation d'une délibération fixant le taux d'un impôt local pour une année peut solliciter du juge de l'impôt que le taux voté l'année précédente soit substitué au taux annulé, à la double condition que ce taux soit légal et inférieur au taux annulé ${ }^{58}$.

Ensuite, à côté de ce contentieux vertical descendant, il en existe également un ascendant. Les collectivités territoriales et les EPCI peuvent effectivement défendre leur autonomie ${ }^{59}$ en contestant devant le juge les contrôles opérés par l'État sur la fiscalité locale. Ils peuvent ainsi demander l'engagement de la responsabilité pour faute de l'État du fait des préjudices subis au titre des erreurs commises par ses services dans la gestion des impôts locaux. En effet, pour différentes raisons ${ }^{60}$, et notamment le principe de l'unité de caisse ${ }^{61}$, l'État liquide, recouvre et vérifie ces impositions. Ces tâches, qui ne sont pas gracieuses ${ }^{62}$, lui assurent une maîtrise des impôts locaux. D'ailleurs, cette organisation confère à l'État la qualité de défendeur dans les litiges de première instance introduits par un contribuable et interdit aux entités locales bénéficiaires de l'impôt local d'avoir la qualité de partie $^{63}$ ou même d'intervenant ${ }^{64}$. «En contrepartie» de ce contrôle de la gestion des impôts locaux, les erreurs que l'État commet dans le cadre de cette mission peuvent engager sa responsabilité auprès des entités locales concernées ${ }^{65}$. Les préjudices dont elles peuvent obtenir la réparation tiennent principalement au « fait de ne pas avoir perçu des impôts ou taxes qui auraient dû être mis en recouvrement ${ }^{66}$. Initialement, le juge recherchait une faute d'une exceptionnelle gravité, puis une faute lourde ${ }^{67}$. Aujourd'hui, après l'arrêt Krupa ${ }^{68}$, il se contente d'une faute simple ${ }^{69}$. Ces litiges, qui peuvent faire l'objet d'un référé-provi$\operatorname{sion}^{70}$, obéissent à des règles particulières ${ }^{71}$. Par exemple, l'entité locale doit agir dans le délai de

\footnotetext{
L. Tartour, L'autonomie financière des collectivités territoriales en droit français, Préface de M. Bouvier, LGDJ 2012, BFPF t. 55, p. 133.

${ }^{2}$ C.E., Ass. 28 déc. 2009, Béziers " 1 », n 304802, Lebon p. 509 ; C.E., Sect., 21 mars 2011, Béziers " 2 " $n^{\circ} 304806$, Lebon p. 117 ; C.E., 27 fév. 2015, Béziers « $3 », n^{\circ} 357028$, Lebon p. 661

${ }^{53}$ Art. 72 al. 6 de la Constitution.

${ }^{54}$ C.A.A. de Nantes, 27 oct. 2009, Préfet d'Eure-et-Loire, n 09NT00293.

${ }^{55}$ C.E., Sect., 30 nov. 2015, Préfet délégué de Saint-Barthélemy et de Saint-Martin, n 388299, Lebon p. 612.

${ }^{56}$ C.E., 12 oct. 1992, Commissaire de la République de la Manche, nº s 88053 e. a., Lebon p. 365.

${ }^{57}$ C.E., 30 mars 2009, Communauté d'agglomération Angers Loire Métropole, $n^{\circ}$ 301219, Lebon T. p. 708.

${ }^{58}$ C.E., 26 juin 1996, SARL Rossi Frères, $n^{\circ} 148711$, Lebon p. 249. Cette solution s'inspire de l'art. 1639 A CGI.

${ }^{59}$ B. Faure, «Les litiges contentieux entre l'État et les collectivités territoriales », D.A. 2017, n 4.

${ }^{60} \mathrm{E}$. Moysan, Les compétences financières locales dans le système juridique français, Avant-propos de M. Bouvier, Préface de J.-M. Pontier, LGDJ, 2015, BFPF t. 61, pp. 38-40.

${ }^{61}$ Art. 26 de la loi organique ${ }^{\circ} 2001-662$ du 1er août 2001 relative aux lois de finances ; art. 47 du décret n²0121246 du 7 nov. 2012 relatif à la gestion budgétaire et comptable publique.

62 L'État perçoit $2 \%$ du produit de ces impôts locaux au titre des frais de gestion : art. 1641 CGI.

${ }^{63}$ C.E., Ass., 20 déc. 1985, Commune de Paris, n 38801, Lebon p. 386.

${ }^{64}$ P. Collin, R. Serpentier, "La responsabilité de l'État à l'égard des collectivités locales en matière fiscale », BJCL 2013, p. 513

${ }^{65}$ E. Moysan, Thèse préc., p. 40

${ }^{66}$ C.E., 16 juill. 2014, Ministre du Budget, $n^{\circ}$ 361570, Lebon p. 220

${ }^{67}$ E. Moysan, Thèse préc., p. 40, note $n^{\circ} 135$

${ }^{68}$ C.E., Sect., 21 mars 2011, n 306225, Lebon p. 101.

${ }^{69}$ C.E., 16 nov. 2011, Commune de Cherbourg-Octeville, n 344621, Lebon T. p. 874

${ }^{70}$ C.E., 31 déc. 2008, Commune de Chateaudouble, $n^{\circ} 305144$.

${ }^{71}$ V. not. : M. Kernéis-Cardinet, "La responsabilité de l'administration fiscale à l'égard des collectivités territoriales », RDP 2019, p. 479
} 
o s s i er

$>$ La fiscalité locale : regards comparatifs Colloque de Lyon 10 octobre 2019

${ }^{72}$ C.E., 16 juill. 2014, Ministre du Budget, $n^{\circ} 361570$, préc.

${ }^{73}$ T.A. de Versailles, 29 nov. 2016, Commune de SaintGermain-en-Laye, $n^{\circ} \mathrm{s}$ 1302230 e. a.

${ }^{74}$ T.A. de Rouen, 16 déc. 2004, Communauté d'agglomération Seine-Eure $n^{\circ}$ 021505, JCP-A 2005, $n^{\circ} 1094$.

${ }^{75}$ C.E., Sect., 21 janv. 1983, Commune de Bastia, n 19933 , Lebon p. 22.

${ }^{76}$ C.E., 15 avril 2005, Ministre de l'Économie, des Finances et de l'Industrie, $n^{\circ} 274855$, Lebon T. p. 760

${ }^{77}$ C.E., $1^{\text {er oct. } 1993, \text { Commune }}$ de Trédaniel, no 115873 , Lebon p. 257.

${ }^{78}$ Art. 5 de la loi n ${ }^{\circ} 2017-1837$ du 30 déc. 2017 de finances pour 2018.

${ }^{79}$ Par ex., à propos d'une réforme de la taxe professionnelle : C.C. 29 déc. 2005, Loi de finances pour 2006, n 2005-530 DC, $\S .93$ et s.

${ }^{80}$ C.C., 29 déc. 2009, Loi de finances pour 2010, $n^{\circ} 2009$ 599 DC, §. 64

${ }^{81}$ Art. $72-2$ de la Constitution.

${ }^{82}$ C.C., 29 juill. 2004, Loi organique relative à l'autonomie financière des collectivités territoriales, $n^{\circ}$ 2004-500 DC, §. 10.

${ }^{83}$ Par ex. : C.C., 29 déc. 2005, Loi de finances pour 2006, n²005-530 DC, préc., §. 96

${ }^{84}$ L'autonomie financière peut être invoquée dans le cadre de l'art. 61-1 de la Constitution: C.C., 29 juin 2012, Départements de la Seine-Saint-Denis et du Var $n^{\circ}$ s 2012-255 QPC e. a., §. 9 .

${ }^{85} \mathrm{C}$. Jerretie, A. Viala, Mission flash sur la réforme des institutions, préc., p. 64

${ }^{86} \mathrm{P}$. de Montalivet, " QPC et droit des collectivités territoriales », AJDA 2016 p. 586.

${ }^{87}$ Loi n $2006-823$ du 10 juill. 2006 autorisant l'approbation de cette Charte ; décret $n^{\circ}$ 2007-679 du 3 mai 2007 portant publication de cette Charte.

${ }^{88}$ Pour les al. 2 et 4 de I'art. 9 : C.E., 26 juill. 2011 Département de la Seine Saint-Denis, $n^{\circ} 340041$ Lebon T. prescription quadriennale et doit indiquer à l'État dans le délai de reprise de l'imposition locale qu'il a commis une erreur dans l'évaluation des bases d'imposition ${ }^{72}$.

Ce régime de responsabilité a été étendu aux hypothèses dans lesquelles, d'une part, la doctrine de l'administration fiscale conduit à une réduction injustifiée du produit des impôts locaux $^{73}$, d'autre part, les services de l'État ne communiquent pas ${ }^{74}$ ou mal ${ }^{75}$ aux entités locales les informations nécessaires au vote des taux des impositions locales. Cependant, l'État est fondé à réduire les attributions mensuelles d'impôts locaux pour ne pas qu'elles excèdent le montant

des rôles homologués s'il a excessivement évalué le montant prévisionnel des bases de ces impositions en fonction duquel l'entité a voté les taux de ces prélèvements ${ }^{76}$. Ce montant n'est effectivement qu'une indication que les assemblées délibérantes ne sont pas tenues de suivre ${ }^{77}$.

Ces contentieux traditionnels de la fiscalité locale sont donc nombreux. Après tout, ils sont le fruit de la multiplication par la décentralisation des relations fiscales entre personnes publiques et des litiges qui peuvent en résulter. Mais, parallèlement, les tensions nées des dynamiques de recentralisation s'amplifient.

\section{La multiplication des contentieux nés des recentralisations de la fiscalité locale}

Du point de vue des entités locales, la recentralisation de la fiscalité locale s'assimile à une perte de pouvoir fiscal. Par conséquent, elles se mobilisent devant le juge pour combattre cette dépossession. Ainsi, ce dernier se trouve saisi de contentieux verticaux ascendants contre la recentralisation de la fiscalité locale au sein de l'État (A.) et des $\mathrm{EPCl}$ (B.).

\section{A. Les contentieux contre la recentra- lisation étatique de la fiscalité locale}

La recentralisation de la fiscalité locale la plus évidente est celle qui se fait au profit de l'État. Elle peut être contestée par les entités locales de deux manières. En premier lieu, les collectivités territoriales peuvent critiquer les lois qui organisent cette étatisation. En effet, le législateur est l'acteur principal de cette dynamique, ainsi que l'illustre en dernier lieu le dégrèvement d'office massif de la taxe d'habitation ${ }^{78}$. Tout d'abord, les collectivités territoriales peuvent critiquer devant le Conseil constitutionnel la constitutionnalité de ces lois au regard de l'atteinte portée à leur autonomie financière. Par le passé, cette contestation était exclusivement indirecte : elle était introduite devant le Conseil constitutionnel par les parlementaires ${ }^{79}$. Mais, compte tenu de la jurisprudence du Conseil, elle était vouée à l'échec. En effet, en l'absence de reconnaissance constitutionnelle de l'autonomie fiscale, le moyen tiré de l'inconstitutionnalité de la suppression du pouvoir de modulation du taux d'une imposition locale est inopérant ${ }^{80}$. De plus, le contrôle de la part déterminante ${ }^{81}$ des ressources fiscales des collectivités territoriales dans l'ensemble de leurs ressources est décevant. En effet, le Conseil admet que ces ressources propres soient constituées des impositions locales et nationales affectées aux collectivités territoriales ${ }^{82}$. En outre, le contrôle de constitutionnalité qu'il opère en la matière réserve une marge de manœuvre importante au législateur ${ }^{83}$.

Aujourd'hui, la contestation des lois mettant en cause la fiscalité locale peut être directe avec la question prioritaire de constitutionnalité (QPC) que les collectivités territoriales introduisent elles-mêmes ${ }^{84}$. D'ailleurs, elles se sont pleinement saisies de cette "nouvelle arme contentieuse $»^{85}$ en matière de fiscalité locale. Mais l'espoir suscité par la QPC a "déçu » ${ }^{86}$ puisque les collectivités territoriales $n$ 'ont jamais obtenu gain de cause par cette voie. Les carences du contrôle de constitutionnalité a priori ont effectivement été transposées à la QPC.

La jurisprudence constitutionnelle est donc favorable à la recentralisation de la fiscalité locale entre les mains de l'État. Face à ce constat, les collectivités locales ont pu considérer la Charte européenne de l'autonomie locale de 1985 et le contrôle de conventionalité comme une échappatoire pour contester les dispositions législatives contraignant leur pouvoir fiscal. Celui-ci est après tout protégé par l'alinéa 3 de l'article 9 de cette Charte qui a été régulièrement introduite dans l'ordre juridique français ${ }^{87}$. Mais la jurisprudence ne s'est pas encore prononcée sur l'invocabilité de ces stipulations ${ }^{88}$. Si leur rédaction laisse penser qu'elles sont d'effet direct, l'appréciation des juridictions administratives ne sera en 
tout état de cause sans doute pas différente de celle du Conseil constitutionnel.

En second lieu, les collectivités territoriales et les EPCl peuvent contester les dispositifs de péréquation verticale. En effet, si les dotations qui leur sont versées par l'État corrigent les inégalités de potentiel fiscal, elles participent aussi à la recentralisation de la fiscalité locale lorsqu'elles compensent la perte de produit fiscal provenant d'un allègement des impositions locales décidé par l'État ${ }^{89}$. Or, les collectivités territoriales et les $\mathrm{EPCl}$ n'ont aucune maîtrise sur ces dotations. Par conséquent, ces entités locales peuvent contester la constitutionnalité des lois qui les diminuent ${ }^{90}$ ou les substituent à des impôts locaux ${ }^{91}$. Mais le contrôle limité de la dénaturation de la libre administration des collectivités territoriales opéré par le Conseil constitutionne ${ }^{92}$ n'entraîne que peu de censures ${ }^{93}$. Dès lors, elles critiquent de plus en plus souvent devant le juge administratif l'absence d'éligibilité à des dotations ${ }^{94}$, leur montant ${ }^{95}$, ou sollicitent le versement de sommes supplémentaires ${ }^{96}$. Les recours en responsabilité pour faute de l'État se multiplient également en réaction à la faiblesse du contrôle opéré par le Conseil constitutionnel. Les fondements invoqués sont divers ${ }^{97}$ : les collectivités et les EPCl peuvent solliciter la réparation de préjudices découlant de la minoration erronée de la dotation ${ }^{98}$ ou de l'absence de compensation intégrale d'allègements de fiscalité locale ${ }^{99}$. Les collectivités territoriales pourraient aussi théoriquement introduire des référés-liberté, la libre administration étant une liberté fondamentale ${ }^{100}$.

\section{B. Les contentieux contre la recentra- lisation de la fiscalité locale}

La recentralisation de la fiscalité locale se fait également au bénéfice des $\mathrm{EPCl}$ à fiscalité propre et au détriment des communes. Celles-ci peuvent agir contre « la disparition » de leur pouvoir fiscal ${ }^{101}$. Tout d'abord, elles peuvent frontalement demander l'annulation de l'arrêté préfectoral créant une intercommunalité à fiscalité propre et les intégrant de force dans son périmètre. En effet, ces EPCl peuvent par exemple instaurer un régime de contribution économique territoriale unique. Or, la création de cette structure permet à " des communes pauvres de se saisir de la richesse d'une commune voisine sur le territoire de laquelle est situé un grand équipement industriel [...] » ${ }^{102}$. Les communes à fort potentiel fiscal peuvent en contester la création ou solliciter l'annulation des délibérations de l'assemblée intercommunale instaurant à son bénéfice un régime de fiscalité additionnelle, unique ${ }^{103}$ ou de zone $e^{104}$, ou fixant les taux des taxes intercommunales ${ }^{105}$.

De manière moins radicale, les communes demandent de plus en plus l'annulation des délibérations intercommunales fixant les montants des attributions de compensation qui leur sont versées en échange du transfert à l'EPCI du droit de percevoir la contribution économique territoriale unique ${ }^{106}$. En effet, ces attributions sont des dépenses intercommunales obligatoires dont le montant annuel doit correspondre au produit de l'impôt perçu jusque-là par la commune diminué du coût net des charges transférées ${ }^{107}$.

Enfin, certaines communes ont sollicité la condamnation de l'EPCl dont elles sont membres à les indemniser des préjudices financiers qu'elles estiment avoir subis en participant au financement de services publics financés par ailleurs par la fiscalité locale qu'elles ont transférée à I'EPCl ${ }^{108}$. Inversement, des intercommunalités ont pu reprocher à des communes membres d'avoir instauré des taxes facultatives dont l'assiette était proche de leur fiscalité intercommunale $e^{109}$

\section{Conclusion}

Avec la décentralisation, les relations entre personnes publiques ayant trait à la fiscalité locale se sont donc "juridictionnalisées » ${ }^{110}$. Les dynamiques de recentralisation ont confirmé ce phénomène qui devrait encore s'affermir avec les actuelles réformes de la fiscalité locale. Ainsi, certaines entités locales contesteront sans doute

${ }^{89}$ M. Bouvier, «Impôts locaux », Jurisclasseur Collectivités territoriales 2018, n²500, ns 110 et s.

${ }^{90}$ C.C., 29 déc. 2014, Loi de finances pour 2015, n²014-707 DC, §. 23.

${ }^{91}$ C.C., 29 déc. 1998, Loi de finances pour 1999, n 98-405 DC, §. 50.

${ }^{92}$ C.C., 29 juin 2012, Départements de la Seine-Saint-Denis et du Var, nº 2012-255 QPC e. a., préc., §. 7

${ }^{93}$ V. cependant : C.C., 14 juin 2013, Communauté de communes Mont d'Or Azergues, n 2013-323 QPC, §. 10.

${ }^{94}$ Par ex. : C.E., 4 juill. 1997, Commune de Trappes, nº 165155.

${ }^{95}$ C.E., 19 nov. 2008, Communauté d'agglomération Évry Val d'Essonne, n² 297438, Lebon T. p. 628.

${ }^{96}$ C.E., 18 oct. 2000, Ministre de l'Économie, des Finances et de I'Industrie, n 209324, Lebon p. 428.

${ }^{97}$ E. Oliva, "La responsabilité de l'État en matière de transferts aux collectivités territoriales 》, BJCL 2013, p. 501.

${ }^{98}$ C.E., 9 mars 2018, Communauté de communes du pays roussillonnais, $n^{\circ}$ 405355, Lebon T. p. 532.

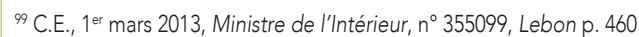

${ }^{100}$ C.E., Sect., 18 janv. 2001, Commune de Venelles, $n^{\circ} 229247$, Lebon p. 18.

${ }^{101}$ B. Faure, op. cit., pp. 401-402.

102 L. Touvet, concl. sur C.E., 2 oct. 1996, Commune de Poulainville e. a., ns 159256 e. a., AJDA 1996, p. 1022.

103 T.A. de Pau, 14 juin 2011, Commune de Tarnos, $n^{\circ} 0902610$

${ }^{104}$ C.A.A. de Bordeaux, $1^{\text {er }}$ avril 2008, Communauté de communes du canton de Navarrenx, nº 06BX00998.

${ }^{105}$ T.A. d'Amiens, 16 juin 2003, Commune de Montataire, $n^{\circ} 031175$; JCP-A 2003, $n^{\circ} 2133$, note J. Moreau.

${ }^{106}$ C.E., 9 mai 2005, Communauté de communes du bassin de Lons-le-Saunier, $n^{\circ} 264551$, Lebon T. p. 724

${ }^{107}$ C.A.A. de Douai, 9 avril 2019, Commune d'Halluin, n’ 16DA01649.

${ }^{108}$ T.A. de Grenoble, 31 oct. 2018, Commune de Pont de Cheruy, n 1606589

${ }^{109}$ C.A.A. de Douai, 9 avril 2019, Commune d'Halluin, n 16DA01649.

110 L. Janicot, «Les conflits entre collectivités territoriales », D.A. 2017, $n^{\circ} 5$. 
Dos sier

$>$ La fiscalité locale : regards comparatifs Colloque de Lyon 10 octobre 2019 les « reprises financières » qui leur seront infligées sur les douzièmes qui leur sont versés mensuellement si elles ne respectent pas les contrats conclus avec l'État pour maîtriser leurs dépenses de fonctionnement ${ }^{111}$. Pourtant, I'analyse des dé- cisions citées dans cette étude démontre que, si I'issue des procès traditionnels de la fiscalité locale est partagée, le sort des instances relatives à la recentralisation favorise surtout cette dynamique.
11 Art. 29 V de la loi n 2018 32 du 22 janv. 2018 de programmation des finances publiques pour les années

2018 à 2022. L'instruction ministérielle du 16 mars 2018 relative à l'application de ces dispositions mentionne d'ailleurs d'éventuels litiges (p. 20). De plus, il faut noter que des contentieux sont déjà survenus s'agissant de la conclusion de ces conventions (par ex., à propos de la contestation d'un arrêté notifiant le niveau maximal annuel de dépenses réelles de fonctionnement T.A. de Montpellier, 18 déc. 2018, Département de

I'Hérault, n 1805903). 